\title{
SIGNAL PROCESSING IN PARTIAL DISCHARGE MEASUREMENT
}

\section{Josef Vedral, Martin Kř́̌̌}

Czech Technical University, Faculty of Electrical Engineering, Department of Measurement, Technická 2, 16627 Prague 6, Czech Republic ( $\triangle$ vedral@fel.cvut.cz, +420 22435 2182, martikriz@gmail.com)

\begin{abstract}
The paper presents traditional analogue and digital techniques in partial discharge measurement. The discharge current pulses are measured from high voltage machines like power plant generators and transformers. The presented modern digital techniques are suitable for all measurements where impulse energy will be calculated.
\end{abstract}

(C) 2010 Polish Academy of Sciences. All rights reserved

\section{Introduction}

Partial Discharge (PD) is a phenomenon in high voltage insulation systems. This phenomenon is a discharge in a void or cavity in an insulation layer. The discharge does not break the full mass of the dielectric layer and does not affect the momentary dielectric strength and momentary breakdown voltage of the dielectric layer. The dielectric strength and breakdown voltage of the dielectric layer are affected and lowered by subsequent PD activities. These long-term PD activities will be monitored and the results will be used for diagnostic purposes and for repairs planning.

There are a lot of non-electric methods for PD monitoring like chemical and optical analysis [1], thermovision, RF power spectral evaluation [2], ultrasonic [3, 4] and [5]. One of sophisticated and standardized methods is the "galvanic method" [6, 7]. The method is based on measurement of voltage pulses on machine terminals. This method is suitable for on-line diagnostics, because it is not necessary to insert any special probe or sensor inside machine windings or use any specialized equipment. The method uses standard high voltage components for coupling.

The key parameter for partial discharge monitoring is apparent charge. The charge is called "apparent charge" because the real charge is inaccessible inside machine windings or insulation layers. The PD meters are calibrated with partial discharge calibrators in parallel with machine terminals - the readout of the PD meters corresponds only with "apparent charge" not with the real charge inside the electric machine. The relation between partial discharge and apparent charge is discussed in [1] and [13].

The typical input circuit of discharge meters is shown in Fig. 1.

A capacitance divider is used for high voltage decoupling. The division ratio is defined by $C_{1}=1 \mathrm{nF}, C_{2}=150 \mathrm{nF}, L=2.5 \mathrm{mH}$ and damping resistor $R=1 \mathrm{k} \Omega$. The frequency bandwidth is defined in international standard from $30 \mathrm{kHz}$ to $500 \mathrm{kHz}$ [1]:

$$
p \approx \frac{C_{1}}{C_{1}+C_{2}} .
$$

In this case the division ratio is $1: 151$ and the resonant frequency is $450 \mathrm{MHz}$.

Voltage and current waves in the dielectric mass are illustrated on Fig. 2. 


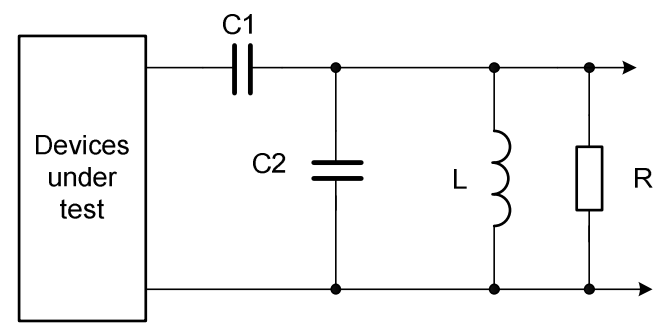

Fig. 1. Standard input circuit of discharge meter.

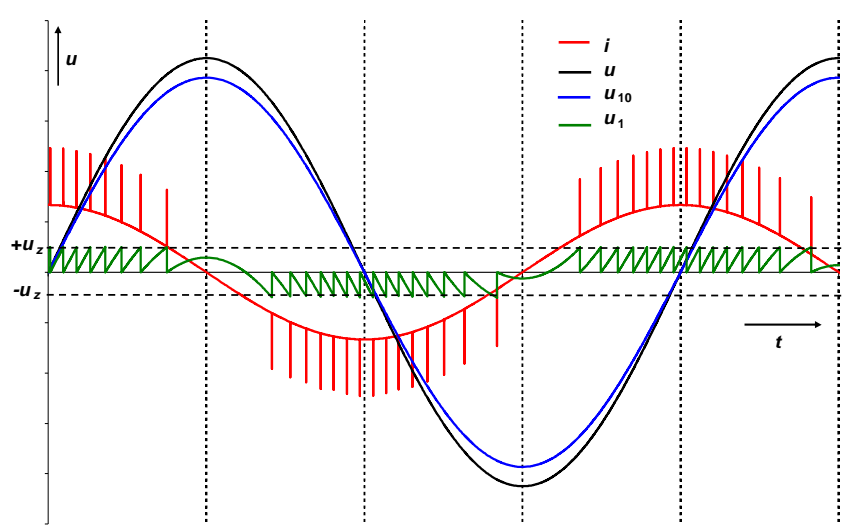

Fig. 2. Time plot of driving and void voltage, current trough the dielectric mass.

Voltage $u$ is the main driving voltage of the dielectric mass. The voltage $u_{10}$ is the voltage on a void in the insulation dielectric layer, without a discharge. The voltage with partial discharge in void $u_{1}$ illustrates the discharge if the ignition voltage $u_{\mathrm{z}}$ is reached. Because the stored energy in void capacitance is discharged, the void will be charged again to the ignition voltage. The current from the power source $i$ has spikes corresponding with the discharge events.

The current spikes are measured and displayed indirectly, by the voltage decreasing pulses on main machine terminals, with consequence of the internal impedance of the machine power source. The discharge events transformed to the voltage pulses are separated by the input circuit from high voltage and low-frequency components.

The input circuit will be designed carefully, because it will separate high voltage $6 \mathrm{kV} / 50$ $\mathrm{Hz}$ and higher voltages from sensitive semiconductor circuits. The separated signal will be integrated or filtrated for evaluation of apparent charge.

\section{Partial discharge frequency spectrum}

The standardized partial discharge impulse [1] has a rising time $T_{1}$ up to $60 \mathrm{~ns}$ and exponential fall time $T_{2}$ to half of the maximum, about $120 \mathrm{~ns}$, Fig. 3.

The charge is measured as the amplitude of the voltage or current pulse. In the standard is defined that the charge is impulse shape independent in a predefined frequency band. The 
frequency band boundaries come from praxis and are standardized. The band-pass filtration can be interpreted as integration.

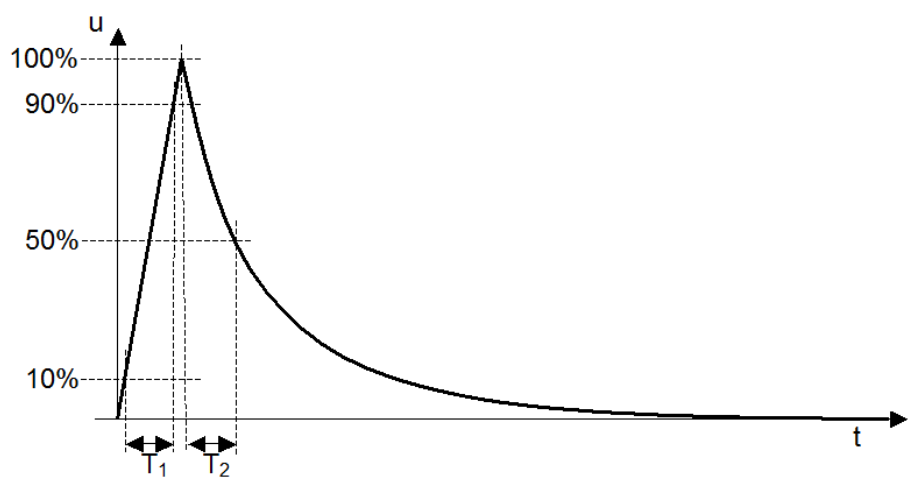

Fig. 3. Partial discharge impulse.

\section{Improved partial discharge measurement techniques}

The major improvement of the traditional PD measurement chain [8] is the use of a logarithmic amplification circuit $[10,11]$ for dynamic range compression and the analog-todigital converter (ADC) for data capturing, see Fig. 4.

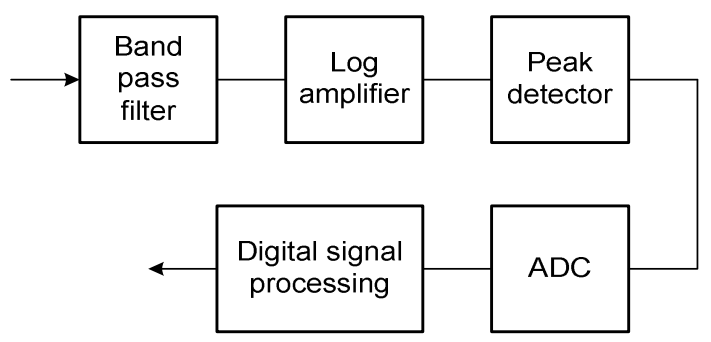

Fig. 4. Improved partial discharge meter with logarithmic amplifier peak detector and ADC.

The PD pulses are frequency limited in a band-pass passive filter. The dynamic range of the incoming signal is compressed in the logarithmic amplifier and maximal values are captured by the peak detector. The captured values are digitized by the ADC and processed in the digital processing circuit [12]. The sampling frequency is not in relation with the input signal. The maximum of the input signal is stored in a peak detector and the detector is zeroed on demand [9]. In the presented PD meter samples, the peak detector is zeroed 256 times in a mains power period. The sampling frequency is $12800 \mathrm{~Hz}$ for $50 \mathrm{~Hz}$ mains voltage frequency. Also the 10-time higher frequency is used for software synchronization purposes with the external signal. The synchronization is useful for localization of a partial discharge source in electric machine windings $[14,15]$.

\section{Fully digital discharge measurement techniques}

The digital techniques are based on using a high-precision and high-speed ADC and digital filters. Two basic approaches can be recognized: digitalizing of the full spectrum of the 
impulse signal with rising times in the nanosecond range with wideband harmonic product to the gigahertz range and digitizing a narrowband signal in boundaries defined in the international standard [1]. The partial discharge charge will be computed in two ways: computing the maximal value in a narrowband signal and computing the area from the digitized wideband signal.

\section{Partial discharge system with charge computed from the impulse area}

The fully digital PD measurement chain is very simple: it is only an ADC with antialiasing filter and a following digital signal processing circuit inside a field-programmable gate array (FPGA) or other specialized circuit, Fig. 5.

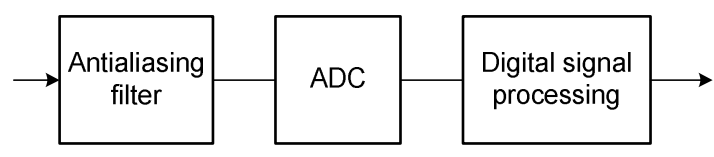

Fig. 5. Fully digital wideband partial discharge meter.

The simplicity of the chain has only one problem with the wideband-signal nature, because the rising edges of the signals range from several nanoseconds for solid dielectric systems and several tens of nanoseconds for liquid isolating systems. It means the ADC will digitize a signal in the range of half a gigahertz. There are limits in the dynamic range and/or in requirements for incoming signal edges. The physical realization of this type of measurement chains are really challenges to electronic circuit design. The major sources of uncertainties in the fully-digital signal chain are uncertainties of the passive antialiasing filter, ADC uncertainties like a gain and offset error, influences of integral nonlinearity, effective number of bits, non-correlated sampling frequency with PD pulses and errors in digital signal processing like rounding are discussed in [12]. The dominant source of the uncertainty is uncertainty from non-correlated sampling frequency with the PD pulse events.

\subsection{Uncertainty of area computation from non-correlated sampling frequency}

The sampling during A/D conversion and the following computation of the pulse area one of the uncertainty sources from non-correlated sampling frequency with PD event; see Fig. 6.

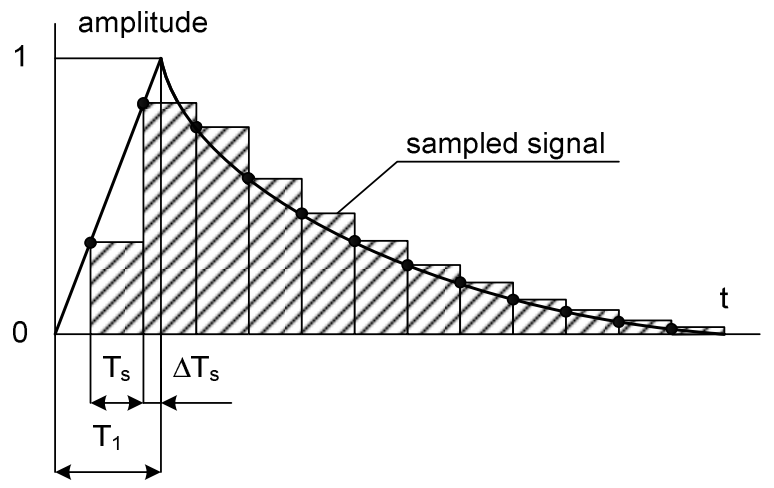

Fig. 6. Charge uncertainty from non correlated sampling. 
This kind of uncertainty can be computed as a variation between the computed ideal area by the continuous integration $S_{\text {int }}$ and by the summation $S_{\text {sum }}$ of the ADC sampled data. For a simple case pulse with linear rise to the maximal value in time $T_{\mathrm{r}}=T_{1} / 0.8$ (in case of linear rising edge) and exponential fall, constant $\tau$ and sampling frequency $f_{\mathrm{s}}$, with delay-ofmaximal-value from sampling time $\Delta T_{\mathrm{s}}$ and its relative value $\delta T_{\mathrm{s}}$, the uncertainty can be computed from the equations (2) and (3):

$$
\begin{array}{r}
S_{\mathrm{int}}=\frac{1}{T_{r}} \int_{0}^{T_{r}} t d t+\int_{t_{r}}^{\infty} e^{-\frac{t-T_{r}}{\tau}} d t=\frac{T_{r}}{2}+\tau, \\
S_{\text {sum }}=\frac{T_{s}^{2}}{2 T_{r}}\left[M^{2}+3 M-2 \delta T_{s}(M-1)\right]+\frac{T_{s} e^{\frac{\delta T_{s} T_{s}+(R-1)}{\tau}}}{1-e^{-\frac{T_{s}}{\tau}}},
\end{array}
$$

where $M$ is an integer part of division $T_{r} / T_{s}$ and $R$ is the decimal fraction of division $T_{r} / T_{s}$. The error is computed as the difference between $S_{\text {int }}$ and $S_{\text {sum }}$, Fig. 7.

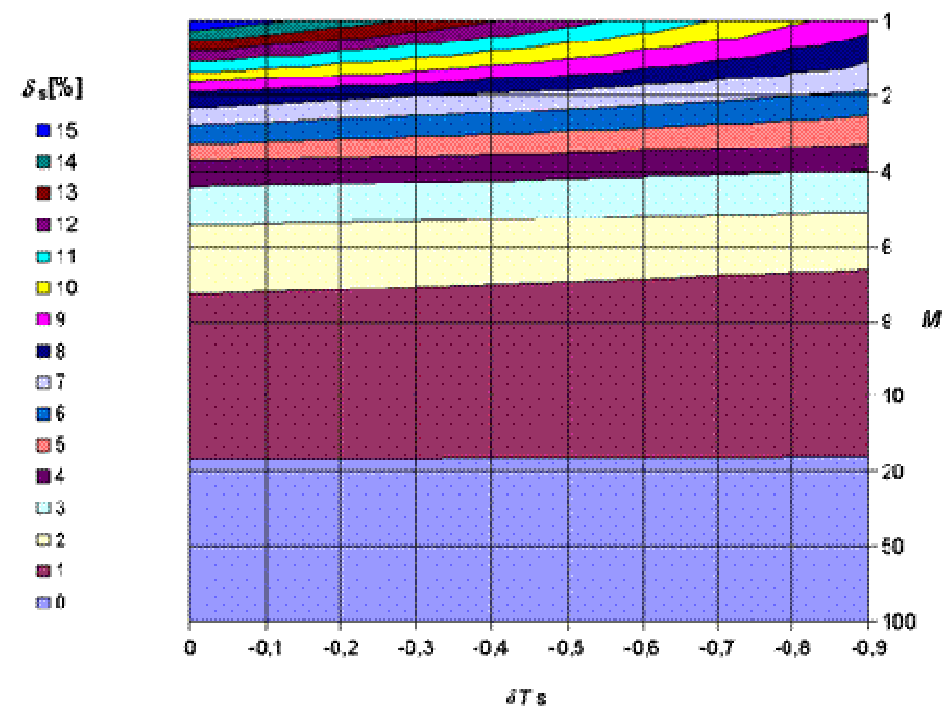

Fig. 7. Chart of charge relative error $\delta_{s}$ on ADC sampling offset $\delta T_{s}$ and rate $M$.

\subsection{Uncertainty of peak value recognition from lathed sampling}

The amplitude of the PD event will be computed in case that software peak detection will be used. In this case another source of uncertainty can be identified. The maximal difference between the peak value and non-correlated sampled value of PD meter input with sampling period $T_{s}$ is in relation (4) with impulse rise time $T_{r}$, impulse upper frequency $f_{h}$, full scale of $\operatorname{ADC} F S_{A D}$ :

$$
\frac{d u}{d t} \max _{\max }=\frac{0.8 F S_{A D}}{T_{r}}=\frac{0.8 F S_{A D} f_{h}}{0.35}=2.29 F S_{A D} f_{h} .
$$

The B type uncertainty can be computed from (5): 


$$
u_{B A D M}=\frac{\Delta_{A D M}}{2 \sqrt{3}}=\frac{2.29 F S_{A D}}{2 \sqrt{3}} \frac{f_{h}}{f_{s}}=0.66 F S_{A D} \frac{f_{h}}{f_{s}} .
$$

The standard uncertainty $u_{B A D M}$ represents the difference between the sampled peak value of the impulse and actual peak value of the impulse in relation to the highest impulse frequency $f_{h}$ and sampling frequency $f_{s}$ of $\mathrm{ADC}$.

The value $2 \sqrt{3}$ comes from type B uncertainty definition; $\Delta_{A D M}$ has a uniform distribution in the interval of $\Delta_{A D M}$.

\section{Hardware design}

For development of the PD meter [11] based on an improved analog technique with a logarithmic amplifier, the wideband (DC- $350 \mathrm{MHz}$ ) full bridge rectifying logarithmic $(88 \mathrm{~dB})$ amplifier AD8307 from Analog devices is used. The controlling microprocessor is the 8051-based Analog Devices ADUC814 with precision 12 bit, $5 \mu \mathrm{sec}$ ADC. The meter has a rugged construction with a surge arrester combined with clamping diodes at the input and galvanically isolated RS232 communication line with heavy-duty transient suppressors at its output. The digital PD meters will use in the future fast ADCs like a Texas Instruments ADS5560 for digitizing a partial frequency spectrum or an ADS5463 or better for wideband digitalization.

\section{Results}

For evaluation purposes the hardware of a PD meter based on improved analogue technique was developed [10,11] and [12]. For further research the fully digital PD meters are pre-evaluated with state-of-the-art monolithic integrated circuits. However the parameters of the digital PD meter are only computed and not proved in praxis. The traditional PD meter is published only for reference.

The transfer characteristic of a PD meter with logarithmic amplifier is shown in Fig. 8.

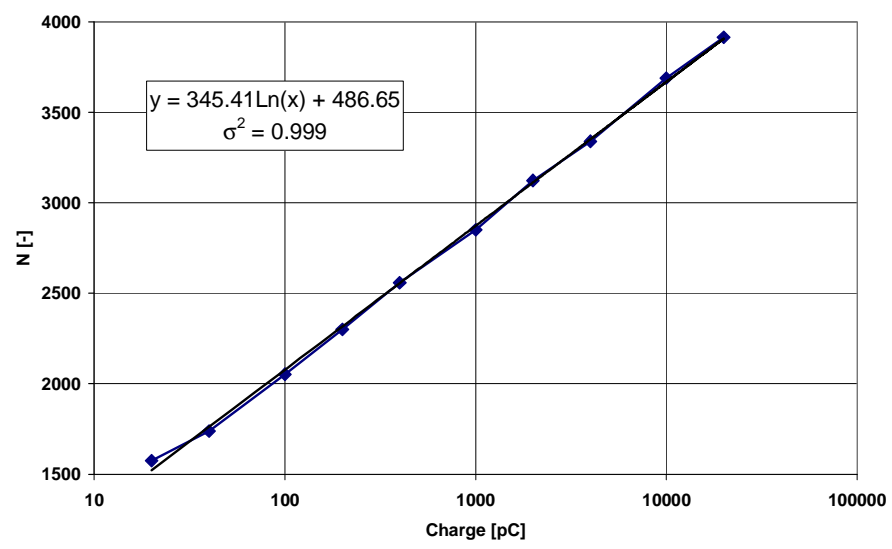

Fig. 8. Partial discharge meter transfer characteristic.

The major improvement is the absence of attenuating switching matrix for range changing and online communication with a master computing system.

The panel of the virtual user interface is shown in Fig. 9. The software controls the PD 
meter, provides calibration, two alarm levels control and has an interface to an expert system based on rules and a neural network for PD activity evaluation.

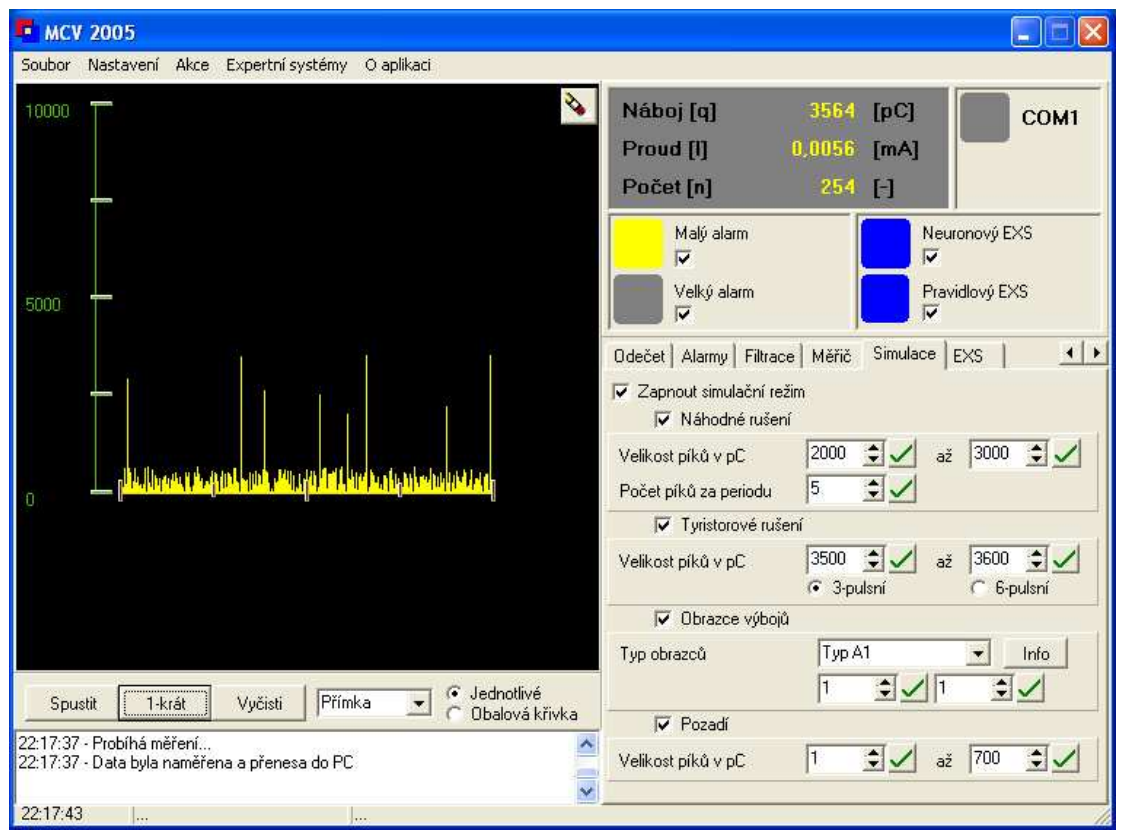

Fig. 9. Partial discharge meter controlling software.

The major parameters of the PD meters based on the described four signal chains are summarized in Table 1.

Table 1. Basic PD meters parameters.

\begin{tabular}{|c|c|c|c|c|}
\hline \multirow{2}{*}{} & \multicolumn{4}{|c|}{ PD meters chain } \\
\cline { 2 - 5 } & \multicolumn{2}{|c|}{ Analog signal processing } & \multicolumn{2}{c|}{ Digital signal processing } \\
\hline Parameter & $\begin{array}{c}\text { Linear } \\
\text { processing }\end{array}$ & $\begin{array}{c}\text { Logarithmic } \\
\text { processing }\end{array}$ & Full BW & Partial BW \\
\hline $\begin{array}{c}\text { ADC sampling } \\
\text { frequency }\end{array}$ & $128 \mathrm{kSa} / \mathrm{s}$ & $128 \mathrm{kSa} / \mathrm{s}$ & $500 \mathrm{Msa} / \mathrm{s}$ & $500 \mathrm{Msa} / \mathrm{s}$ \\
\hline ADC resolution & $12 \mathrm{bit}$ & $12 \mathrm{bit}$ & $16 \mathrm{bit}$ & $16 \mathrm{bit}$ \\
\hline Nonlinearity & $0,15 \%$ of FS & $0,2 \%$ of FS & $0,03 \%$ of FS & $0,05 \%$ of FS \\
\hline Dynamic range & $87 \mathrm{~dB}$ & $69 \mathrm{~dB}$ & $75 \mathrm{~dB}$ & $93 \mathrm{~dB}$ \\
\hline
\end{tabular}

The table contains two fully digital signal chains, one digitizing the whole signal spectrum and one for digitizing only the range described in [2]. There are some limitations in the frequency range due to the commercial availability of a monolithic integrated circuit with high resolution and high sampling frequency.

In the table there are also two analog signal chains with digitalization - one without a logarithmic amplifier and the same chain improved with a logarithmic amplifier for easy comparison. The improvement in dynamic range is dramatic and opens the door for many measurements including self online diagnostics for rotary machines in power plants with predicting diagnostic systems. 


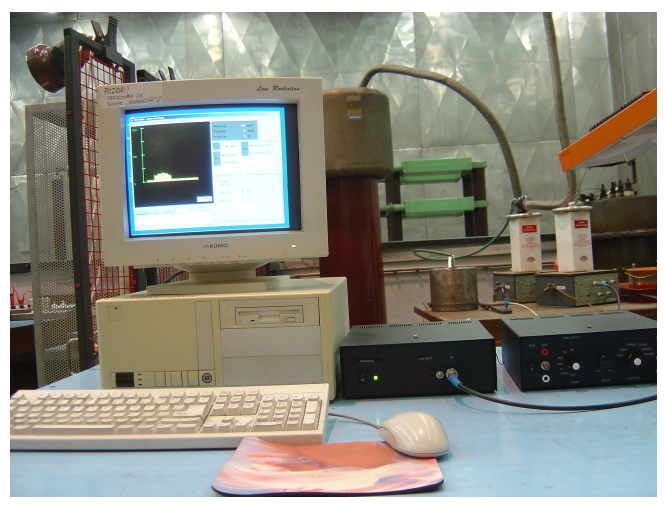

Fig. 10. Workspace overview in high voltage laboratory.

\section{Comparison}

Four signal chains are compared. First, a traditional signal chain with a peak detection circuit and low-speed ADC. Second, a signal chain with logarithmic amplifier and peak detection circuit with low-speed ADC. Third, a fully-digital signal chain with narrowband digitalization and finally a fourth signal chain with wideband digitalization of the PD signals. The major results are presented in the following table.

The traditional analogue signal chains are limited by the dynamic range of the peak detection circuits, but the electronic part of the design is relative simple.

The digital signal chains, specially the signal chain with digitalization of the full wideband signal is a complex digital signal chain and the parameters of the digital filters acquisition algorithms can be changed because many computing parameters are only variables in the software.

All four signals chains can be used for on-line diagnostic measurements and can save a lot of time and sources in maintenance of the high voltage units.

\section{Conclusion}

The article presents traditional and modern ways in on-line diagnostic measurement of the state of the high voltage insulation system from electronic and data acquisition point of view. Four measurement chains are analyzed - two analog-based and two digital-based. Comparisons are made for four analyzed chains with real components.

Future ways in on-line acquisition of the PD activities research are presented. The area computing technique can be used in many other impulse measurements.

This technique is not able to recognize the shape of an impulse; other additional techniques will be used like discrimination of pulses with long/short rise/fall time, packed pulses (pileup rejection) etc.

\section{Acknowledgments}

This project is supported by the research program No. MSM6840770015 "Research of Methods and Systems for Measurement of Physical Quantities and Measured Data Processing" of the CTU in Prague sponsored by the Ministry of Education, Youth and Sports of the Czech Republic. 


\section{References}

[1] R. Schwarz, M. Muhr, S. Pack: "Partial discharge detection in oil with optical methods". IEEE International Conference ICDL, Coimbra, Portugal. June 2005, pp. 245-248.

[2] D. Dapeng, T.L. Qian Yong, W. Jiang, H. Chengjun, Z. Yi, J. Xiuchen: "Experimental study and feature extraction on UHF partial discharge detection for typical model in air". International Conference on Condition Monitoring and Diagnosis, 2008, 21-24 April 2008, pp. 1040-1043.

[3] P. Kundu, N.K. Kishore, A.K. Sinha: "Simulation and Analysis of Acoustic Wave Propagation due to Partial Discharge Activity". IEEE Conference on Electrical Insulation and Dielectric Phenomena, 15-18 Oct. 2006, pp. 607-610.

[4] L. Yanqing, L. Fangcheng, X. Baoan, Ch. Zhiye: "A new method using ultrasonic for partial discharge pattern recognition”. International Conference on Power System Technology, 13-17 Oct. 2002, pp.10041007.

[5] C. Macia-Sanahuja, H. Lamela-Rivera: "Wavelet analysis of partial discharges acoustic waves obtained using an optical fibre interferometric sensor for transformer applications". IEEE International Symposium of Industrial Electronics, June 2003, pp. 1071-1076.

[6] D. Koenig, Y. Narayana Rao: Partial Discharges in Electrical Power Apparatus. VDE-Verlag; Germany, 1998.

[7] EN 60270: High-voltage test techniques - Partial discharge measurements. Prague 2001, Czech Normalised Institut, Cat. Number 63384.

[8] F.J. Wester, E. Guilski, J.J. Smit: "Detection of Partial Discharges at Different AC Voltage Stresses in Power Cables". IEEE Electrical Insulation Magazine, vol. 23, no. 4, 2007, pp. 28-43.

[9] A. Cavallini, G.C. Montanari, M. Olivieri, F. Puletti: "Diagnostic evaluations of electrical apparatus by a novel partial discharge measurement system". International Conference on Power System Technology, vol. 2, 2004, pp. 1689-1693.

[10] M. Kříž, J. Vedral: "Quantification of Partial Discharges Charge”. 4th International Conference on Measurement Measurement, Slovak Academy of Sciences, 2003, pp. 407-410.

[11] M. Kř́̌ž, J. Vedral: "Preprocessing Circuits for Partial Discharges Measurement”. International Workshop on ADC Modelling and Testing, Universita degli Studi di Perugia, 2003, pp. 201-203.

[12] M. Kř́̌ž, J. Vedral: "Partial Discharge Meter". International Conference of Applied Electronics 2004, University of West Bohemia FEE, Pilsen, 2004, pp. 124-126.

[13] M. Kř́̌ž, J. Vedral: "Partial discharge measurement systems and methods optimalization". PhD. Thesis, CTU FEE, Prague 2009.

[14]K. Záliš: Partial discharges in insulating systems of electrical machines. Publishing House ACADEMIA, Prague 2005.

[15] K. Záliš, L. Karasová-Beranová: "Problems with Partial Discharge Measurement Disturbance Elimination". Electric Power Conference EPVE, Brno, FEKT, 2008, pp.1-6. CD-ROM.

[16] M. Kriz, J. Vedral: "Digital signal processing in partial discharge measurement". IEEE Inteligent Data Acqusition Systems IDACS, 2009, Rende, Cosenza, Italy. CD-ROM. 